\title{
Impacts of Plant Presence on Formaldehyde Levels in an Office
}

\author{
Isra Abu Zayed ${ }^{1}$, Bassam Abu Hijleh², Hanan Taleb ${ }^{2}$ \\ ${ }^{1}$ Faculty of Fine Arts and Design, American University in the Emirates \\ UAE \\ isra.abuzayed@aue.ae \\ ${ }^{2}$ Faculty of Engineering and IT, British University in Dubai \\ $\mathrm{UAE}$ \\ bassam.abuhijleh@buid.ac.ae; hanan.taleb@buid.ac.ae
}

\begin{abstract}
The toxin formaldehyde has been associated with muscle weakness, itchy eyes and skin and is considered a carcinogen. The toxin poses a risk to the occupants of a space when found at high concentrations. Plant life has been long associated with cleaning air and improving indoor air quality levels, so this study will look at the impact of plant life on formaldehyde levels within an existing office in the United Arab Emirates. Through the selection of a plant that has been proven to reduce formaldehyde levels (i.e. Corn Cane), the study will determine the extent of the ability of the plant to reduce formaldehyde levels in an existing office setting. Three spaces within an office were selected; labelled as space A, B and C. The plants were included within these spaces, each with a different space/plant ratio. The plants remained within the space for one month before a secondary round of testing was conducted. Results showed that plants were able to reduce the highest amount of formaldehyde levels when introduced at the highest plant/space ratio of $3.75 \mathrm{~m} 2 /$ plant (Space B). This allowed the plants to be more effective within their space. When equated, there was a total drop of $4 \%$ in formaldehyde levels within space B.
\end{abstract}

Keywords: Indoor Air Quality, Formaldehyde, Toxins, United Arab Emirates, Plant Life

\section{Introduction}

The United Arab Emirates (UAE) is a country that has emerged from the sands of the Gulf desert in fewer than fifty years and is now a world-renowned country with metropolitan cities that have evolved greatly over the last four decades. This success was achieved through innovative design of buildings which were completed in a timely fashion. In the early 1970s, structures were built with the aim of maximising internal thermal comfort, increasing efficiency and cutting costs. Architects and engineers alike worked towards insulating these spaces in order to ensure that the occupants would be comfortable. This, in turn, led to occupants spending the majority of their time indoors; whether at home, at the work place or during their leisure time. The comfort levels that were achieved encouraged people to begin to depend entirely on interior spaces, which began a dependence on HVAC systems to clean and filter indoor air. Furthermore in these sealed interiors, there was an addition of toxins to the space. Toxins were - and still continue to be - emitted within interior spaces through insulation, paints and finishes, furniture and cleaning products [1]. This toxic build-up within spaces became a source of illness and put people at risk of contracting diverse diseases. Toxins such as VOCs - chemicals containing carbon and hydrogen - have been proven to have adverse health effects if found within a space [2]. One common type of VOC is formaldehyde ( $\mathrm{HCHO})$, which has been associated with muscle weakness, itchy eyes and skin and is considered a carcinogen. This toxin poses a risk to occupants' of any space when found at high concentrations [2].

Indoor Air Quality (IAQ) has now become a serious concern. The risks which inhabitants are facing if indoor air quality is of poor quality are severe. As the UAE continues its progress towards an innovative future it is vital to consider the wellbeing of occupants through healthy indoor air quality. Past research has shown a connection between the presence of plant life within a space and the ability to reduce and absorb such toxins. One of the most commonly known methods of plants aiding the reduction of toxins is carbon dioxide and oxygen exchange. Recent research has connected the presence of plant life within interiors with a means to connect with nature. Plant life has the ability to help stabilise certain toxins within a space. The presence of plant life can also be directly linked to the reduced amount of airborne moulds and bacteria within a space. In order to determine the depth at which plant life may impact on toxins within the interior, more research 
is needed. This project will involve conducting a study to determine the ability of plant life to impact on formaldehyde levels in an office interior setting in the UAE.

\subsection{Literature Review}

Formaldehyde is a colourless gas at room temperature and can be found within our atmosphere. It is emitted though natural and pollutant sources and can be found naturally within the environment through forest fires and volcanoes. In addition, through pollution it is introduced into the atmosphere through power plants and incinerators [3].

According to the WHO [3] formaldehyde indoors is a toxin which needs to be monitored. Large concentrations of this toxin found within a space and exposed to humans through inhalation can have adverse health effects on the occupants. Formaldehyde can be introduced to building interiors through material emissions and combustible activities such as smoking, cooking or lighting candles. In addition it is also used as a resin and preservatives in many manufacturing processes. Also mentioned by the WHO is that some of the factors which determine the rate at which formaldehyde is emitted into the environment are furniture age and density. New furniture emits toxins at a higher rate resulting in an increase in formaldehyde levels, whereas old or used furniture has allowed emissions to fade over the years [3].

According to the EPA, everyone is exposed to some levels of formaldehyde from off-gasses emitted by products. Products which are produced from composite woods have the highest rate of formaldehyde emissions. This is due to the high amount of formaldehyde resin added to the woods. In 2010 President Obama signed legislation to set and police a standard for the amount of emitted formaldehyde from these products [4]. Raw et al. conducted a large scale study which surveyed 876 homes located in the United Kingdom in order to determine factors which impact on the increase of formaldehyde levels in a space. It was concluded that the age of the building was an important aspect of determining formaldehyde concentrations. More specifically it was the age of the building walls and floor coverings [4]. Orwell et al. conducted a lab experiment to determine the ability of a plant and its root (microorganisms) to reduce TVOC. The labconducted study looked at several houseplants which were exposed to a variety of levels of TVOC (ranging from 0.2 to $100 \mathrm{ppm}$ ). It concluded that plants and the microorganisms in roots were able to self-regulate in order to absorb a larger quantity of TVOC as their presence within the space increased [5]. Another study conducted in 2004, saw seven potted plants tested for their ability to remove the toxin benzene from an enclosed chamber conducted through laboratory research. It was determined that the plants were able to remove high doses of benzene from within the chamber within twenty four hours [6].

Wood et al. [7] looked at three common house plants and their ability to reduce common airborne VOCs. It was concluded that plant life has the capability to act as a sustainable bio-filtration system within a space. The air cleaning capability along with the psychological and aesthetic benefits of the plant make it an ideal solution to incorporate within a home or office. Kim [8] conducted a lab study to identify the ability of the 'Fatsia Japanica' plant to reduce formaldehyde levels within a laboratory setting. It was concluded that the plant was able to reduce formaldehyde levels at a greater rate during the night hours due to microorganisms found within the roots.

In the UAE, people spend more than ninety per cent of their time indoors [9]. While indoors, there is complete reliance on HVAC systems. These systems, a means to alleviate the environment of toxins, are not always reliable. Office workers in the UAE spend a minimum of eight hours a day indoors. Within these office spaces, the entire airflow system is conducted by the HVAC system. Employees are left with no choice but to depend on HVAC to achieve thermal comfort, with some buildings and institutions going as far as preventing people from opening windows. In addition to this the majority of office furniture is created from MDF (desks and shelving units) which has been shown to emit the highest concentrations of formaldehyde [10]. This complete dependence on the HVAC system, along with previous research which raised concerns in relation to formaldehyde, creates a research gap relating to chemical testing in an office setting to find a possible solution.

The aim of the study is to determine the impacts of a selected plant on formaldehyde levels within an existing office interior in Dubai. In addition, the study will investigate whether reduction of formaldehyde levels has any effect on employee moral or perceived health and also whether the presence of plant life has an effect on employee moral or perceived health.

\section{Methodology}

The experiment will be conducted in an office space set in the Ras Al Khor Industrial Area 1, located in Dubai, UAE. The office is within a large warehouse with half of the facility is utilized as storage and the other half being an office area 
consisting of two floors and a total of eight work spaces. There are twenty one people employed by the company and four of the employees spend the majority of their day out of the office. The remaining seventeen employees work primarily at a desk and behind a computer. The main services provided are logistics and clearing.

Three spaces where selected to accommodate the experiment. The first, Space A, is located on the lowest floor. There are three work stations within the space and four storage cabinets. The space is also adjacent to the staircase and there are a total of three people occupying this space. The area contains one window which allows for natural lighting and is to remain closed for the entire during of the experiment. The second space selected, Space B, is located on the first floor. It is a room that contains three accountants and has one window that is fixed and overlooks another space within the office. The space does not have any natural lighting and there is no possibility of opening a window during the one month period. The room is quite dense and contains three desks within a tight space. The third space is also located on the third floor, is the largest of the three and contains four workstations with four regular occupants. Referred to as Space C, it also contains a window that allows for natural lighting and will remain closed for the duration of the experiment.

Three methods of data collection are required to obtain the necessary data from each of the three allocated spaces (A, $\mathrm{B}$ and $\mathrm{C})$. The first is the collection and recording of temperature and humidity within the space. As mentioned by Godish [10] the presence of the formaldehyde toxin decreases as temperature and humidity drop. We need to monitor the temperature and humidity of the formaldehyde levels in order to ensure that the formaldehyde readings attained are not altered due to changed weather conditions. It is important to ensure that they are collected at approximately the same time during the day in order to avoid any alterations which might occur due to change in sun exposure leading to changes in temperature or humidity. The second method of data collection is determining the formaldehyde levels, which are best estimated within an enclosed space. The equipment will be placed on a desk within the centre of each room and left for a total of nine hours. The first hour of testing is not included within the results as the testing equipment will be in the process of stabilising. The remaining eight hours of testing will test continuously over ten minute intervals and will record the results in order to allow reading of the results at a later date. This method of data collection is adapted as it is the method advised by the Dubai Government Green Building Regulations and Specifications Handbook [11]. The third method of data collection is a survey created to collect feedback from the occupants about their perceived health and productivity. Occupants of the space answered the survey anonymously but are asked to share any health issues which they have recently faced which might alter the survey results. All occupants of the space are male and between the ages of 28-50. The survey consisted of five symptoms which the occupants were asked to rate on a scale of 'never', 'once in a while' and 'almost daily'.

For the first method of data collection, Gray Wolf IQ- 610 was utilised to record temperature and relative humidity. The instrument is handheld and can be easily moved from one space to another to conduct spot measurements. The second method utilised the Gray Wolf Formaldehyde Measurement device. The device is to be set up on a desktop within the space and left uninterrupted for the desired amount of hours. The third method was the survey circulated to employees found within the spaces. The survey requested that people sharer feedback on five different questions relating to perceived heath and productivity. They were asked to rate the frequency at which they experienced these aspects during the last two weeks. The reason behind associating the survey with the last two weeks is that once the survey is conducted a second time it becomes a review of the last period of time in which the plants had the greatest effect on the inhabitants. This was the last two weeks of the overall month period in which the plants were added to the space.

The plant selected for the experiment is the 'Corn Cane' plant. This is a plant that is usually found within residential homes. The plant does not take up too much space and does not cause any irritation to the occupants around it. The edges of the plants are not sharp and it has not been found to be poisonous. Plant maintenance and upkeep is very simple, it requires a low amount of exposure to sunlight and a low amount of water to survive. The selection of a plant which has low maintenance requirements aids the ease at which the office management can take on the addition of plants and ensures that it is not a burden. Additionally, it is important to select a plant which can confidently live within the space. The longevity of the plant ensures that offices feel that the amount of money invested is worth the return.

The corn cane plant will be introduced to each of the spaces (A, B and C). Each space will receive three plants, resulting in a total of nine plants added to the offices. When determining the amount of plants required, it was important to select a number of plants that is acceptable for an office setting. Having too many plants would be a distraction to the employees and can have a negative effect on concentration and worker efficiency. On the other hand, having too few plants can lead to a lack of results. Although all of the spaces are somewhat enclosed and have three to four people within the spaces, there is still a variation in the plant to space ratio achieved. 
- Space A: Addition of three plants to the pace resulted in a ratio of $5.25 \mathrm{~m}^{2} / \mathrm{plant}$

- Space B: Addition of three plants to the smallest space resulted in the highest space to plant ratio of $3.75 \mathrm{~m}^{2} / \mathrm{plant}$

- Space C: Addition of the three plants to the largest space resulted in the lowest space to plant ratio of $7.166 \mathrm{~m}^{2} / \mathrm{plant}^{2}$

When conducting an experiment in a place of work there are certain associated limitations. The first is the dynamic nature of the office setting as occupants of the space move around throughout the office over the hours of the day. Additionally visitors, business meetings and deliveries are made, which keeps the space environment dynamic. In addition to this, although occupants of the space were made aware of the experiment in hand, it is difficult to ensure that they do not take part in activities during the span of a month that might impact on the results; activities such as opening a window or spraying perfume. The occupants were made aware of the research being conducted, so it is a challenge to ensure that the survey results shared are accurate and not swayed by their perception of what the result should be. Human psychology plays a factor in a need to give results which adhere to the goals of the study. The last limitation faced was an attempt to maintain temperature and relative humidity within the space. Temperature within the space was set to twenty three degrees during the entire month of the experiment in order to ensure a constant temperature throughout.

One of the main strategies in sustainable design is the orientation of a building. This will help to optimise solar and wind aspects which need to be targeted and used as an advantage. The UAE marine wind assessment shows prevailing winds coming in from the north-west end, so it is important to establish an entry point for the wind to enter the space.

\section{Results}

Table 1 shows the results in relation to the spaces selected and that a notable impact has been made on Space B formaldehyde levels. The characteristics of Space B provided the ideal situation in which the plants were able to impact on the space at the greatest rate; characteristics such as being a smaller and more controlled space with a high density of occupants and formaldehyde emissions.

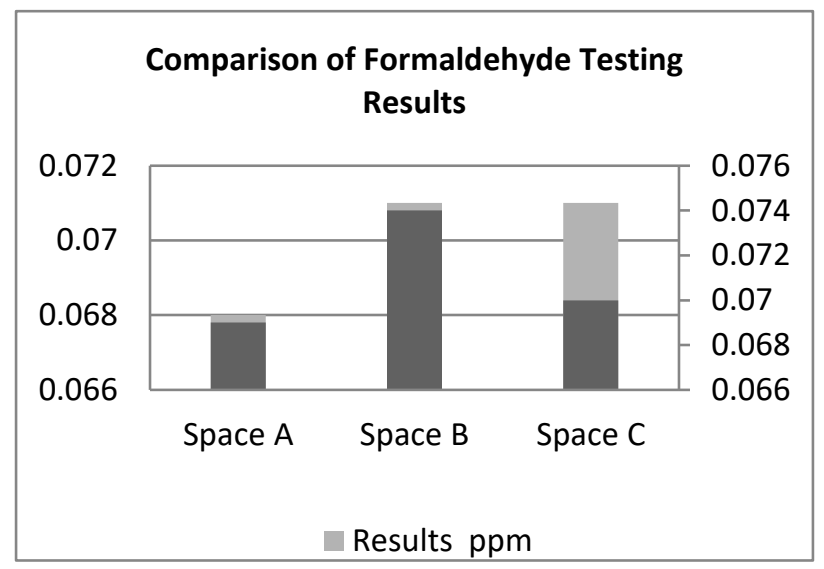

Fig. 1: Overall Formaldehyde Results.

Plant life was able to absorb the greatest amount of formaldehyde levels when the greatest amount was present. They were able to help reduce the levels of formaldehyde from $0.074 \mathrm{ppm}$ to $0.071 \mathrm{ppm}$, a total reduction of four per cent. The two remaining spaces saw minimal changes occurring to their formaldehyde levels. The lack of change may well be associated with three factors. The first is low initial formaldehyde levels; low formaldehyde emissions give the plants little to absorb. The second is changes in temperature and humidity which could have particularly affected space $\mathrm{C}$ - where the largest change in relative humidity occurred. The thirst aspect is the size of the spaces which created a low space-to-plant ratio.

The results of the survey show a drastic improvement. The initial survey results showed that the majority of the employees had suffered some of the perceived health symptoms over the previous two weeks. Although the symptoms were not drastic - example: daily headache - such symptoms were still present. As seen in the initial results (Fig.2), every one of the employees suffered a headache within the two weeks prior to the date in which the initial survey was conducted. The majority of headaches were noted to be regular occurrences, in that they were present several times a week. Headaches 
were the most frequently mentioned in terms of the perceived health aspect of the survey. The other perceived health questions, itchy eyes or skin and a sore, dry throat and or wheezing, received minimal association. When looking at perceived personal productivity, the majority of the workers felt difficulty concentrating. A rate of eighty and ninety per cent mentioned a feeling of fatigue at some time during the last two weeks.

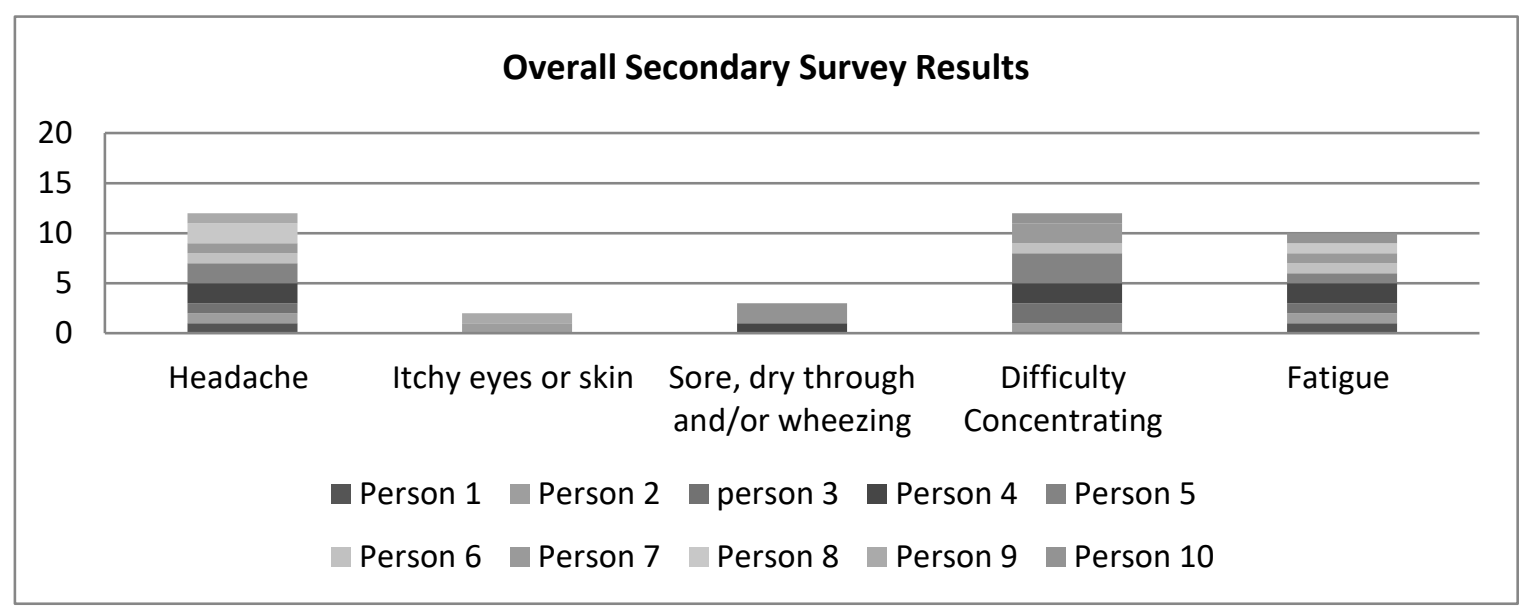

Fig. 2: Overall Secondary Survey Results.

After the corn cane plant was introduced to the space (Fig.2), it is noted that eighty per cent of the staff had experienced a minor headache at some point during the previous two weeks. This showed a drop of thirty six per cent in headache symptoms. There was a similar drop of thirty three per cent in the category of itchy eyes and skin. As for sore throat, cough and or wheezing, this dropped by half. When looking at overall results related to the perceived health of the employees, there is an overwhelming improvement. When compared with the results on perceived productivity, a drop of twenty per cent was achieved. As for the general feeling of fatigue, that was reduced by a total of forty four per cent.

The above results and improvement in employee perceived health and productivity far outweigh the results which were achieved by the decrease in formaldehyde concentrations. This leads to the conclusion that the plant life itself is having a positive effect on the employees. The psychological impacts of plants and plant life have been shown in previous research. Smith and Pitt [12] showed how the ability of plant life to improve in-office moral far outweighed the results achieved of the plants reducing the amount of toxins present.

\section{Conclusion}

The initial aim of this study was to look at the impact of plant life on formaldehyde levels within an office in the UAE. Formaldehyde is a toxic VOC that is present in our environment. It is produced naturally through volcanic eruptions and any combustive activity. The levels of formaldehyde have, however, increased over the last forty years due to its inclusion as a resin within furniture, finishes and insulation. This high presence of formaldehyde within interior spaces poses a health risk to the occupants involved. Initial data collection results for the office space that was selected showed that levels of formaldehyde within the office were relatively high but still not reaching the maximum concentration of $0.08 \mathrm{ppm}$. The survey results, however, showed that occupants of the space suffered headaches regularly, an inability to concentrate and a general feeling of fatigue.

Plant life was introduced for a period of one month, after which the data collection process began again. The formaldehyde levels showed a decrease of four per cent when the plant life was introduced to the space with the highest level of formaldehyde (space B), as well as the highest space-to-plant rations of $3.75 \mathrm{~m}^{2} /$ plant. The space was also an enclosed space that had the most controlled environment, leading to the conclusion that the results of formaldehyde reduction from a space increase when the presence of formaldehyde is greater. Additionally, formaldehyde levels are reduced at a more optimised rate when the space-to-plant ratio is increased.

When looking at the overall survey results collected, there is a significant improvement in office morale. Employees felt an improved sense of perceived health and productivity. This is paralleled by the results which show a decrease in the symptoms previously felt within the space. The results also showed a twenty per cent decrease in an inability to 
concentrate within the space as well as a forty four per cent decrease in feelings of fatigue while at the office. The space with the most improved survey results was space $\mathrm{C}$. Employees within that space experienced an improvement across all of the questions posed over the survey, which proved to be an interesting result, as space $\mathrm{C}$ had the least improved level of formaldehyde. In fact, space $\mathrm{C}$ saw an increase of formaldehyde levels by $1.4 \%$. The lack of connection between formaldehyde level improvement and survey results shows that perceived health and productivity levels are not connected to formaldehyde levels. To the contrary, perceived health and productivity levels were directly associated with introducing plant life into the space.

These results show the extent to which plant life was able to impact on the occupants of the space whether or not formaldehyde levels were decreased. The impact of plant life on the space was far greater than that of the reduced formaldehyde levels. The psychological impacts of the presence of plant life on the occupants of the space gave them an improved perception of their health and productivity.

\section{References}

[1] M. Mendell, "Indoor Residential Chemical Emissions as Risk Factors for Respiratory and Allergic Effects in Children: A Review," Indoor Air Journal, vol. 17, pp. 259-277, 2007.

[2] K. Kaitlin, "Dangerous Decor: Consumer Knowledge of Health Risks within Interior Spaces," SJSU ScholarWorks, 2011.

[3] WHO, "Guidelines for Indoor Air Quality: Selected Pollutants," World Health Organization Regional Office for Europe, pp. 142-142, 2010.

[4] G. J. Raw, S. K. D. Coward, V. M. Brown, D. R. Crump, "Exposure to Air Pollutants in English Homes," Journal of Exposure Analysis and Environmental Epidemiology, vol. 14, pp. 85-94, 2004.

[5] R. L. Orwell, R. A. Wood, M. D. Burchett, J. Tarran, F. Torpy, The potted-plant microcosm substantially reduces indoor air VOC pollution: II. Laboratory study, Water Air Soil, 2006.

[6] R. Wood, "Plants Improve Air Quality and Cut Energy Consumption, Reports International Scientific Conference," Facilities, vol. 18, no. 3-4, 2000.

[7] R. A. Wood, R. L. Orwell, J. Tarran, F. Torphy, M. Burchett, "Potted-plant/Growth Media Interactions and Capacities for Removal of Volatiles from Indoor Air," Journal of Horticultural Science \& Biotechnology, vol. 77, no. 1, pp. 120-129, 2002.

[8] S. E. Kim, "Physical Workplace as a Strategic Asset for Improving Performance in Public Organizations," Administration \& Society, vol. 46, no. 5, pp. 496-518, 2014.

[9] Arabian Business, Sick Building Syndrome Fixes, 2007.

[10] T. Godish, Indoor air Pollution Control, IEQ Indoor Plants (n. d.) Benefits of Indoor Plants, 1989.

[11] Dubai Government. Green Building Regulations \& Specifications, 2011.

[12] A. Smith, M. Pitt, "Sustainable Workplaces: Improving Staff Health and Well-being using Plants," Journal of Corporate Real Estate, vol. 11, no. 1, pp. 52-65, 2009.

[13] B. C. Wolverton, J. D. Wolverton, "Plants and Soil Microorganisms," Journal of the Mississippi Academy of Sciences, pp. 11-15, 2000. 